\title{
Joint Constraints on Galactic Diffuse Neutrino Emission from the ANTARES and IceCube Neutrino Telescopes
}

A. Albert ${ }^{1}$, M. André ${ }^{2}$, M. Anghinolfi ${ }^{3}$, M. Ardid ${ }^{4}$, J.-J. Aubert ${ }^{5}$, J. Aublin ${ }^{6}$, T. Avgitas ${ }^{6}$, B. Baret ${ }^{6}$, J. Barrios-Martí ${ }^{7}$, S. Basa ${ }^{8}$, B. Belhorma ${ }^{9}$, V. Bertin ${ }^{5}$, S. Biagi ${ }^{10}$, R. Bormuth ${ }^{11,12}$, J. Boumaaza ${ }^{13}$, S. Bourret ${ }^{6}$, M. C. Bouwhuis ${ }^{11}$, H. Brânzaş ${ }^{14}$, R. Bruijn ${ }^{11,15}$, J. Brunner ${ }^{5}$, J. Busto ${ }^{5}$, A. Capone ${ }^{16,17}$, L. Caramete ${ }^{14}$, J. Carr ${ }^{5}$, S. Celli ${ }^{16,17,18}$, M. Chabab ${ }^{19}$, R. Cherkaoui El Moursli ${ }^{13}$, T. Chiarusi ${ }^{20}$, M. Circella ${ }^{21}$, J. A. B. Coelho ${ }^{6}$, A. Coleiro ${ }^{6,7}$, M. Colomer $^{6,7}$, R. Coniglione ${ }^{10}$, H. Costantini ${ }^{5}$, P. Coyle ${ }^{5}$, A. Creusot $^{6}$, A. F. Díaz ${ }^{22}$, A. Deschamps ${ }^{23}$, C. Distefano ${ }^{10}$, I. Di Palma ${ }^{16,17}$, A. Domi ${ }^{3,24}$, C. Donzaud ${ }^{6,25}$, D. Dornic ${ }^{5}$, D. Drouhin ${ }^{1}$, T. Eberl ${ }^{26}$, I. El Bojaddaini ${ }^{27}$, N. El Khayati ${ }^{13}$, D. Elsässer ${ }^{28}$, A. Enzenhöfer ${ }^{5,26}$, A. Ettahiri ${ }^{13}$, F. Fassi ${ }^{13}$, I. Felis ${ }^{4}$, P. Fermani ${ }^{16,17}$, G. Ferrara ${ }^{10}$, L. Fusco ${ }^{6,29}$, P. Gay ${ }^{6,30}$, H. Glotin ${ }^{31}$, T. Grégoire ${ }^{6}$, R. Gracia Ruiz ${ }^{1}$, K. Graf ${ }^{26}$, S. Hallmann ${ }^{26}$, H. van Haren ${ }^{32}$, A. J. Heijboer ${ }^{11}$, Y. Hello ${ }^{23}$, J. J. Hernández-Rey ${ }^{7}$, J. Höß12 ${ }^{26}$, J. Hofestädt ${ }^{26}$, G. Illuminati ${ }^{7}$, C. W. James ${ }^{26}$, M. de Jong ${ }^{11,12}$, M. Jongen ${ }^{11}$, M. Kadler ${ }^{28}$, O. Kalekin ${ }^{26}$, U. Katz ${ }^{26}$, N. R. Khan-Chowdhury ${ }^{7}$, A. Kouchner ${ }^{6,33}$, M. Kreter ${ }^{28}$, I. Kreykenbohm ${ }^{34}$,

V. Kulikovskiy ${ }^{3,35}$, C. Lachaud ${ }^{6}$, R. Lahmann ${ }^{26}$, D. Lefèvre ${ }^{36,37}$, E. Leonora ${ }^{38}$, G. Levi ${ }^{20,29}$, M. Lotze ${ }^{7}$, S. Loucatos ${ }^{6,39}$, M. Marcelin ${ }^{8}$, A. Margiotta ${ }^{20,29}$, A. Marinelli ${ }^{40,41}$, J. A. Martínez-Mora ${ }^{4}$, R. Mele ${ }^{42,43}$, K. Melis ${ }^{11,44}$, P. Migliozzi ${ }^{42}$, A. Moussa $^{27}$, S. Navas ${ }^{45}$, E. Nezri ${ }^{8}$, A. Nuñez ${ }^{5,8}$, M. Organokov ${ }^{1}$, G. E. Păvălaş ${ }^{14}$, C. Pellegrino ${ }^{20,29}$, P. Piattelli ${ }^{10}$, V. Popa ${ }^{14}$, T. Pradier ${ }^{1}$, L. Quinn $^{5}$, C. Racca $^{46}$, N. Randazzo ${ }^{38}$, G. Riccobene ${ }^{10}$, A. Sánchez-Losa ${ }^{21}$, M. Saldaña ${ }^{4}$, I. Salvadori ${ }^{5}$, D. F. E. Samtleben ${ }^{11,12}$, M. Sanguineti ${ }^{3,24}$, P. Sapienza ${ }^{10}$, F. Schüssle ${ }^{39}$, M. Spurio ${ }^{20,29}$, Th. Stolarczyk ${ }^{39}$, M. Taiuti ${ }^{3,24}$, Y. Tayalati ${ }^{13}$, A. Trovato ${ }^{10}$, B. Vallage ${ }^{6,39}$, V. Van Elewyck ${ }^{6,33}$, F. Versari ${ }^{20,29}$, D. Vivolo ${ }^{42,43}$, J. Wilms ${ }^{34}$, D. Zaborov ${ }^{5}$, J. D. Zornoza ${ }^{7}$, J. Zúñiga ANTARES Collaboration,

and

M. G. Aartsen ${ }^{47}$, M. Ackermann ${ }^{48}$, J. Adams ${ }^{47}$, J. A. Aguilar ${ }^{49}$, M. Ahlers ${ }^{50}$, M. Ahrens ${ }^{51}$, I. Al Samarai ${ }^{52}$, D. Altmann ${ }^{53}$, K. Andeen ${ }^{54}$, T. Anderson ${ }^{55}$, I. Ansseau ${ }^{49}$, G. Anton ${ }^{53}$, C. Argüelles ${ }^{56}$, J. Auffenberg ${ }^{57}$, S. Axani ${ }^{56}$, P. Backes ${ }^{57}$, H. Bagherpour ${ }^{47}$, X. Bai ${ }^{58}$, A. Barbano ${ }^{52}$, J. P. Barron ${ }^{59}$, S. W. Barwick ${ }^{60}$, V. Baum ${ }^{61}$, R. Bay ${ }^{62}$, J. J. Beatty ${ }^{63,64}$, J. Becker Tjus ${ }^{65}$, K.-H. Becker ${ }^{66}$, S. BenZvi ${ }^{67}$, D. Berley ${ }^{68}$, E. Bernardini ${ }^{48}$, D. Z. Besson ${ }^{69}$, G. Binder ${ }^{62,70}$, D. Bindig ${ }^{66}$, E. Blaufuss ${ }^{68}$, S. Blot ${ }^{48}$, C. Bohm ${ }^{51}$, M. Börner ${ }^{71}$, F. Bos ${ }^{65}$, S. Böser ${ }^{61}$, O. Botner ${ }^{72}$, E. Bourbeau ${ }^{50}$, J. Bourbeau ${ }^{73}$, F. Bradascio ${ }^{48}$, J. Braun ${ }^{73}$, M. Brenzke ${ }^{57}$, H.-P. Bretz ${ }^{48}$, S. Bron ${ }^{52}$, J. Brostean-Kaiser ${ }^{48}$, A. Burgman ${ }^{72}$, R. S. Busse ${ }^{73}$, T. Carver ${ }^{52}$, E. Cheung ${ }^{68}$, D. Chirkin ${ }^{73}$, A. Christov ${ }^{52}$,

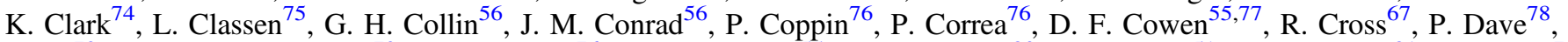
M. Day ${ }^{73}$, J. P. A. M. de André ${ }^{79}$, C. De Clercq ${ }^{76}$, J. J. DeLaunay ${ }^{55}$, H. Dembinski ${ }^{80}$, K. Deoskar ${ }^{51}$, S. De Ridder ${ }^{81}$, P. Desiati ${ }^{73}$,

K. D. de Vries ${ }^{76}$, G. de Wasseige ${ }^{76}$, M. de With ${ }^{82}$, T. DeYoung ${ }^{79}$, J. C. Díaz-Vélez ${ }^{73}$, V. di Lorenzo ${ }^{61}$, H. Dujmovic ${ }^{83}$,

J. P. Dumm ${ }^{51}$, M. Dunkman ${ }^{55}$, E. Dvorak ${ }^{58}$, B. Eberhardt ${ }^{61}$, T. Ehrhardt ${ }^{61}$, B. Eichmann ${ }^{65}$, P. Eller ${ }^{55}$, P. A. Evenson ${ }^{80}$, S. Fahey $^{73}$, A. R. Fazely ${ }^{84}$, J. Felde ${ }^{68}$, K. Filimonov ${ }^{62}$, C. Finley ${ }^{51}$, A. Franckowiak ${ }^{48}$, E. Friedman ${ }^{68}$, A. Fritz ${ }^{61}$, T. K. Gaisser ${ }^{80}$, J. Gallagher ${ }^{85}$,

E. Ganster ${ }^{57}$, L. Gerhardt ${ }^{70}$, K. Ghorbani ${ }^{73}$, W. Giang ${ }^{59}$, T. Glauch ${ }^{86}$, T. Glüsenkamp ${ }^{53}$, A. Goldschmidt ${ }^{70}$, J. G. Gonzalez ${ }^{80}$,

D. Grant ${ }^{59}$, Z. Griffith ${ }^{73}$, C. Haack ${ }^{57}$, A. Hallgren ${ }^{72}$, L. Halve ${ }^{57}$, F. Halzen ${ }^{73}$, K. Hanson ${ }^{73}$, D. Hebecker ${ }^{82}$, D. Heereman ${ }^{49}$,

K. Helbing ${ }^{66}$, R. Hellauer ${ }^{68}$, S. Hickford ${ }^{66}$, J. Hignight ${ }^{79}$, G. C. Hill ${ }^{87}$, K. D. Hoffman ${ }^{68}$, R. Hoffmann ${ }^{66}$, T. Hoinka ${ }^{71}$,

B. Hokanson-Fasig ${ }^{73}$, K. Hoshina ${ }^{73,100}$, F. Huang ${ }^{55}$, M. Huber ${ }^{86}$, K. Hultqvist ${ }^{51}$, M. Hünnefeld ${ }^{71}$, R. Hussain ${ }^{73}$, S. In $^{83}$, N. Iovine $^{49}$, A. Ishihara ${ }^{88}$, E. Jacobi ${ }^{48}$, G. S. Japaridze ${ }^{89}$, M. Jeong ${ }^{83}$, K. Jero ${ }^{73}$, B. J. P. Jones ${ }^{90}$, P. Kalaczynski ${ }^{57}$, W. Kang ${ }^{83}$, A. Kappes ${ }^{75}$, D. Kappesser ${ }^{61}$, T. $\mathrm{Karg}^{48}$, A. Karle ${ }^{73}$, U. Katz ${ }^{53}$, M. Kauer ${ }^{73}$, A. Keivani ${ }^{55}$, J. L. Kelley ${ }^{73}$, A. Kheirandish ${ }^{73}$, J. Kim ${ }^{83}$, T. Kintscher ${ }^{48}$, J. Kiryluk ${ }^{91}$, T. Kittler ${ }^{53}$, S. R. Klein ${ }^{62,70}$, R. Koirala ${ }^{80}$, H. Kolanoski ${ }^{82}$, L. Köpke ${ }^{61}$, C. Kopper ${ }^{59}$, S. Kopper ${ }^{92}$, J. P. Koschinsky ${ }^{57}$, D. J. Koskinen ${ }^{50}$, M. Kowalski ${ }^{48,82}$, K. Kringss ${ }^{86}$, M. Kroll ${ }^{65}$, G. Krückl ${ }^{61}$, S. Kunwar ${ }^{48}$, N. Kurahashi ${ }^{93}$,

A. Kyriacou ${ }^{87}$, M. Labare ${ }^{81}$, J. L. Lanfranchi ${ }^{55}$, M. J. Larson ${ }^{50}$, F. Lauber ${ }^{66}$, K. Leonard ${ }^{73}$, M. Leuermann ${ }^{57}$, Q. R. Liu ${ }^{73}$, E. Lohfink ${ }^{61}$, C. J. Lozano Mariscal ${ }^{75}$, L. Lu ${ }^{88}$, J. Lünemann ${ }^{76}$, W. Luszczak ${ }^{73}$, J. Madsen ${ }^{94}$, G. Maggi ${ }^{76}$, K. B. M. Mahn ${ }^{79}$, Y. Makino ${ }^{88}$, S. Mancina ${ }^{73}$, R. Maruyama ${ }^{95}$, K. Mase ${ }^{88}$, R. Maunu ${ }^{68}$, K. Meagher ${ }^{49}$, M. Medici ${ }^{50}$, M. Meier ${ }^{71}$, T. Menne ${ }^{71}$,

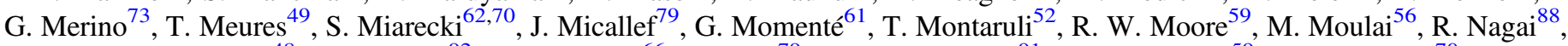
R. Nahnhauer ${ }^{48}$, P. Nakarmi ${ }^{92}$, U. Naumann ${ }^{66}$, G. Neer $^{79}$, H. Niederhausen ${ }^{91}$, S. C. Nowicki ${ }^{59}$, D. R. Nygren ${ }^{70}$,

A. Obertacke Pollmann ${ }^{66}$, A. Olivas ${ }^{68}$, A. O’Murchadha ${ }^{49}$, E. O’Sullivan ${ }^{51}$, T. Palczewski ${ }^{62,70}$, H. Pandya ${ }^{80}$, D. V. Pankova ${ }^{55}$, P. Peiffer ${ }^{61}$, J. A. Pepper ${ }^{92}$, C. Pérez de los $\operatorname{Heros}^{72}$, D. Pieloth ${ }^{71}$, E. Pinat ${ }^{49}$, A. Pizzuto ${ }^{73}$, M. Plum ${ }^{54}$, P. B. Price ${ }^{62}$,

G. T. Przybylski ${ }^{70}$, C. Raab ${ }^{49}$, M. Rameez ${ }^{50}$, L. Rauch ${ }^{48}$, K. Rawlins ${ }^{96}$, I. C. Rea ${ }^{86}$, R. Reimann ${ }^{57}$, B. Relethford ${ }^{93}$, E. Resconi ${ }^{86}$, W. Rhode ${ }^{71}$, M. Richman ${ }^{93}$, S. Robertson ${ }^{87}$, M. Rongen ${ }^{57}$, C. Rott ${ }^{83}$, T. Ruhe ${ }^{71}$, D. Ryckbosch ${ }^{81}$, D. Rysewyk ${ }^{79}$, I. Safa ${ }^{73}$,

S. E. Sanchez Herrera ${ }^{59}$, A. Sandrock ${ }^{71}$, J. Sandroos ${ }^{61}$, M. Santander ${ }^{92}$, S. Sarkar ${ }^{50,97}$, S. Sarkar ${ }^{59}$, K. Satalecka ${ }^{48}$, M. Schaufel ${ }^{57}$, P. Schlunder ${ }^{71}$, T. Schmidt ${ }^{68}$, A. Schneider ${ }^{73}$, S. Schöneberg ${ }^{65}$, L. Schumacher ${ }^{57}$, S. Sclafani ${ }^{93}$, D. Seckel ${ }^{80}$, S. Seunarine ${ }^{94}$, J. Soedingrekso ${ }^{71}$, D. Soldin ${ }^{80}$, M. Song ${ }^{68}$, G. M. Spiczak ${ }^{94}$, C. Spiering ${ }^{48}$, J. Stachurska ${ }^{48}$, M. Stamatikos ${ }^{63}$, T. Stanev ${ }^{80}$,

A. Stasik $^{48}$, R. Stein ${ }^{48}$, J. Stettner ${ }^{57}$, A. Steuer ${ }^{61}$, T. Stezelberger ${ }^{70}$, R. G. Stokstad ${ }^{70}$, A. Stöß1 ${ }^{88}$, N. L. Strotjohann ${ }^{48}$, T. Stuttard ${ }^{50}$, G. W. Sullivan ${ }^{68}$, M. Sutherland ${ }^{63}$, I. Taboada ${ }^{78}$, F. Tenholt ${ }^{65}$, S. Ter-Antonyan ${ }^{84}$, A. Terliuk ${ }^{48}$, S. Tilav ${ }^{80}$, P. A. Toale ${ }^{92}$, M. N. Tobin ${ }^{73}$, C. Tönnis ${ }^{83}$, S. Toscano ${ }^{76}$, D. Tosi ${ }^{73}$, M. Tselengidou ${ }^{53}$, C. F. Tung ${ }^{78}$, A. Turcati ${ }^{86}$, C. F. Turley ${ }^{55}$, B. Ty $^{73}$, E. Unger ${ }^{72}$, M. A. Unland Elorrieta ${ }^{75}$, M. Usner ${ }^{48}$, J. Vandenbroucke ${ }^{73}$, W. Van Driessche ${ }^{81}$, D. van Eijk ${ }^{73}$, N. van Eijndhoven ${ }^{76}$, 
S. Vanheule ${ }^{81}$, J. van Santen ${ }^{48}$, M. Vraeghe ${ }^{81}$, C. Walck ${ }^{51}$, A. Wallace ${ }^{87}$, M. Wallraff ${ }^{57}$, F. D. Wandler ${ }^{59}$, N. Wandkowsky ${ }^{73}$, T. B. Watson ${ }^{90}$, A. Waza ${ }^{57}$, C. Weaver ${ }^{59}$, M. J. Weiss ${ }^{55}$, C. Wendt ${ }^{73}$, J. Werthebach ${ }^{73}$, S. Westerhoff ${ }^{73}$, B. J. Whelan ${ }^{87}$, N. Whitehorn ${ }^{98}$, K. Wiebe ${ }^{61}$, C. H. Wiebusch ${ }^{57}$, L. Wille ${ }^{73}$, D. R. Williams ${ }^{92}$, L. Wills ${ }^{93}$, M. Wolf ${ }^{86}$, J. Wood ${ }^{73}$, T. R. Wood ${ }^{59}$, E. Woolsey ${ }^{59}$, K. Woschnagg ${ }^{62}$, G. Wrede ${ }^{53}$, D. L. Xu ${ }^{73}$, X. W. Xu ${ }^{84}$, Y. Xu ${ }^{91}$, J. P. Yanez ${ }^{59}$, G. Yodh ${ }^{60}$, S. Yoshida $^{88}$, T. Yuan $^{73}$ IceCube Collaboration,

and

D. Gaggero ${ }^{99}$, and D. Grasso ${ }^{40,41}$

${ }^{1}$ Université de Strasbourg, CNRS, IPHC UMR 7178, F-67000 Strasbourg, France; antares.spokeperson@in2p3.fr

${ }^{2}$ Technical University of Catalonia, Laboratory of Applied Bioacoustics, Rambla Exposició, E-08800 Vilanova i la Geltrú, Barcelona, Spain ${ }^{3}$ INFN-Sezione di Genova, Via Dodecaneso 33, I-16146 Genova, Italy

${ }^{4}$ Institut d'Investigació per a la Gestió Integrada de les Zones Costaneres (IGIC) - Universitat Politècnica de València. C/ Paranimf 1, E-46730 Gandia, Spain

${ }^{5}$ Aix Marseille Univ, CNRS/IN2P3, CPPM, Marseille, France

${ }^{6}$ APC, Univ Paris Diderot, CNRS/IN2P3, CEA/Irfu, Obs de Paris, Sorbonne Paris Cité, France

${ }^{7}$ IFIC-Instituto de Física Corpuscular (CSIC-Universitat de València) c/ Catedrático José Beltrán, 2 E-46980 Paterna, Valencia, Spain

${ }^{8}$ LAM-Laboratoire d'Astrophysique de Marseille, Pôle de l'Étoile Site de Château-Gombert, rue Frédéric Joliot-Curie 38, F-13388 Marseille Cedex 13, France

${ }^{9}$ National Center for Energy Sciences and Nuclear Techniques, B.P.1382, R.P.10001 Rabat, Morocco

${ }^{10}$ INFN-Laboratori Nazionali del Sud (LNS), Via S. Sofia 62, I-95123 Catania, Italy

${ }^{11}$ Nikhef, Science Park, Amsterdam, The Netherlands

${ }^{12}$ Huygens-Kamerlingh Onnes Laboratorium, Universiteit Leiden, The Netherlands

${ }^{13}$ University Mohammed V in Rabat, Faculty of Sciences, 4 av. Ibn Battouta, B.P. 1014, R.P. 10000 Rabat, Morocco

${ }^{14}$ Institute of Space Science, RO-077125 Bucharest, Măgurele, Romania

${ }^{15}$ Universiteit van Amsterdam, Instituut voor Hoge-Energie Fysica, Science Park 05, 1098 XG Amsterdam, The Netherlands

${ }^{16}$ INFN-Sezione di Roma, P.le Aldo Moro 2, I-00185 Roma, Italy

${ }^{17}$ Dipartimento di Fisica dell’Università La Sapienza, P.le Aldo Moro 2, I-00185 Roma, Italy

${ }^{18}$ Gran Sasso Science Institude, Viale Francesco Crispi 7, I-00167 L’Aquila, Italy

${ }^{19}$ LPHEA, Faculty of Science-Semlali, Cadi Ayyad University, P.O.B. 2390, Marrakech, Morocco

${ }^{20}$ INFN_Sezione di Bologna, Viale Berti-Pichat 6/2, I-40127 Bologna, Italy

${ }^{21}$ INFN-Sezione di Bari, Via E. Orabona 4, I-70126 Bari, Italy

${ }^{22}$ Department of Computer Architecture and Technology/CITIC, University of Granada, 18071 Granada, Spain

${ }^{23}$ Géoazur, UCA, CNRS, IRD, Observatoire de la Côte d'Azur, Sophia Antipolis, France

${ }^{24}$ Dipartimento di Fisica dell'Università, Via Dodecaneso 33, I-16146 Genova, Italy

${ }^{25}$ Université Paris-Sud, F-91405 Orsay Cedex, France

${ }^{26}$ Friedrich-Alexander-Universität Erlangen-Nürnberg, Erlangen Centre for Astroparticle Physics, Erwin-Rommel-Str. 1, 91058 Erlangen, Germany

${ }^{27}$ University Mohammed I, Laboratory of Physics of Matter and Radiations, B.P.717, Oujda 6000, Morocco

${ }^{28}$ Institut für Theoretische Physik und Astrophysik, Universität Würzburg, Emil-Fischer Str. 31, 97074 Würzburg, Germany

${ }_{29}$ Dipartimento di Fisica e Astronomia dell'Università, Viale Berti Pichat 6/2, 40127 Bologna, Italy

${ }^{30}$ Laboratoire de Physique Corpusculaire, Clermont Université, Université Blaise Pascal, CNRS/IN2P3, BP 10448, F-63000 Clermont-Ferrand, France

LIS, UMR Université de Toulon, Aix Marseille Université, CNRS, F-83041 Toulon, France

${ }^{32}$ Royal Netherlands Institute for Sea Research (NIOZ) and Utrecht University, Landsdiep 4, 1797 SZ 't Horntje (Texel), The Netherlands

${ }^{33}$ Institut Universitaire de France, F-75005 Paris, France

${ }^{34}$ Dr. Remeis-Sternwarte and ECAP, Friedrich-Alexander-Universität Erlangen-Nürnberg, Sternwartstr. 7, 96049 Bamberg, Germany

${ }_{36}^{35}$ Moscow State University, Skobeltsyn Institute of Nuclear Physics, Leninskie gory, 119991 Moscow, Russia

${ }^{36}$ Mediterranean Institute of Oceanography (MIO), Aix-Marseille University, F-13288, Marseille, Cedex 9, France

${ }^{37}$ Université du Sud Toulon-Var, CNRS-INSU/IRD UM 110, F-83957, La Garde Cedex, France

${ }^{38}$ INFN-Laboratori Nazionali del Sud (LNS), Via S. Sofia 64, I-95123 Catania, Italy

${ }^{39}$ Direction des Sciences de la Matière-Institut de recherche sur les lois fondamentales de l'Univers-

Service de Physique des Particules, CEA Saclay, F-91191 Gif-sur-Yvette Cedex, France

${ }^{40}$ INFN-Sezione di Pisa, Largo B. Pontecorvo 3, I-56127 Pisa, Italy

${ }^{41}$ Dipartimento di Fisica dell'Università, Largo B. Pontecorvo 3, I-56127 Pisa, Italy ${ }^{42}$ INFN-Sezione di Napoli, Via Cintia I-80126 Napoli, Italy

${ }^{44}$ Universiteit van Amsterdam, Instituut voor Hoge-Energie Fysica, Science Park 105, 1098 XG Amsterdam, The Netherlands

${ }^{45}$ Dpto. de Física Teórica y del Cosmos \& C.A.F.P.E., University of Granada, E-18071 Granada, Spain

${ }^{46}$ GRPHE-Université de Haute Alsace-Institut universitaire de technologie de Colmar, 34 rue du Grillenbreit BP 50568-68008 Colmar, France

${ }^{47}$ Department of Physics and Astronomy, University of Canterbury, Private Bag 4800, Christchurch, New Zealand; analysis@icecube.wisc.edu

${ }^{48}$ DESY, D-15738 Zeuthen, Germany

${ }^{49}$ Université Libre de Bruxelles, Science Faculty CP230, B-1050 Brussels, Belgium

${ }^{50}$ Niels Bohr Institute, University of Copenhagen, DK-2100 Copenhagen, Denmark

${ }^{51}$ Oskar Klein Centre and Dept. of Physics, Stockholm University, SE-10691 Stockholm, Sweden

${ }^{52}$ Département de physique nucléaire et corpusculaire, Université de Genève, CH-1211 Genève, Switzerland

${ }^{53}$ Erlangen Centre for Astroparticle Physics, Friedrich-Alexander-Universität Erlangen-Nürnberg, D-91058 Erlangen, Germany

${ }^{54}$ Department of Physics, Marquette University, Milwaukee, WI, 53201, USA

${ }^{55}$ Department of Physics, Pennsylvania State University, University Park, PA 16802, USA

${ }^{56}$ Department of Physics, Massachusetts Institute of Technology, Cambridge, MA 02139, USA

${ }^{57}$ III. Physikalisches Institut, RWTH Aachen University, D-52056 Aachen, Germany

${ }^{58}$ Physics Department, South Dakota School of Mines and Technology, Rapid City, SD 57701, USA

${ }^{59}$ Department of Physics, University of Alberta, Edmonton, Alberta, T6G 2E1, Canada

${ }^{60}$ Department of Physics and Astronomy, University of California, Irvine, CA 92697, USA

${ }^{61}$ Institute of Physics, University of Mainz, Staudinger Weg 7, D-55099 Mainz, Germany

${ }^{62}$ Department of Physics, University of California, Berkeley, CA 94720, USA

${ }^{63}$ Department of Physics and Center for Cosmology and Astro-Particle Physics, Ohio State University, Columbus, OH 43210, USA

${ }^{64}$ Department of Astronomy, Ohio State University, Columbus, OH 43210, USA

${ }^{65}$ Fakultät für Physik \& Astronomie, Ruhr-Universität Bochum, D-44780 Bochum, Germany

${ }^{66}$ Department of Physics, University of Wuppertal, D-42119 Wuppertal, Germany 


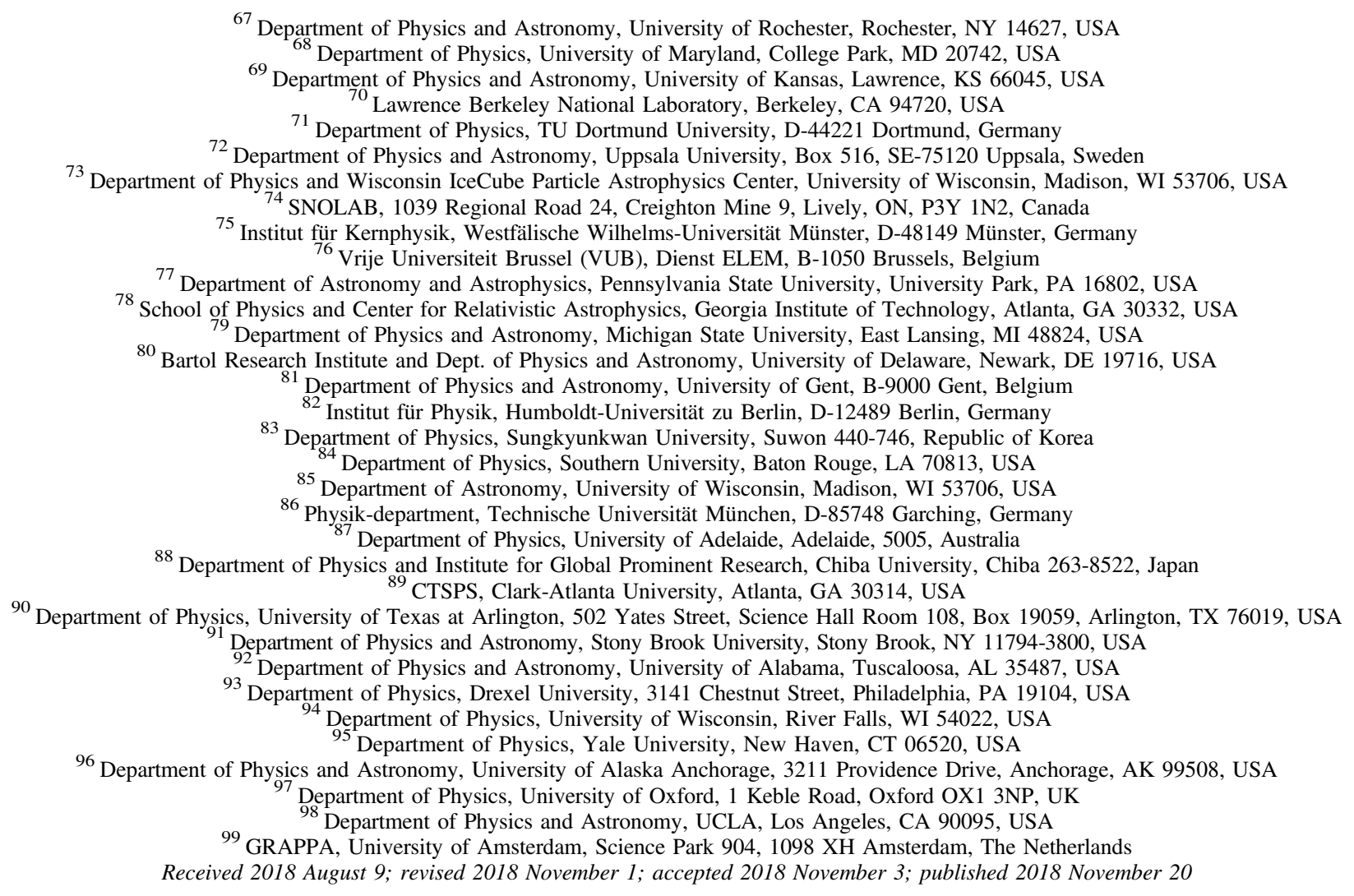

\begin{abstract}
The existence of diffuse Galactic neutrino production is expected from cosmic-ray interactions with Galactic gas and radiation fields. Thus, neutrinos are a unique messenger offering the opportunity to test the products of Galactic cosmic-ray interactions up to energies of hundreds of $\mathrm{TeV}$. Here we present a search for this production using ten years of Astronomy with a Neutrino Telescope and Abyss environmental RESearch (ANTARES) track and shower data, as well as seven years of IceCube track data. The data are combined into a joint likelihood test for neutrino emission according to the $\mathrm{KRA}_{\gamma}$ model assuming a $5 \mathrm{PeV}$ per nucleon Galactic cosmic-ray cutoff. No significant excess is found. As a consequence, the limits presented in this Letter start constraining the model parameter space for Galactic cosmic-ray production and transport.
\end{abstract}

Key words: cosmic rays - diffusion - Galaxy: disk - gamma rays: diffuse background - neutrinos

\section{Introduction}

A diffuse Galactic neutrino emission is expected from cosmic-ray (CR) interactions with interstellar gas and radiation fields. These interactions are also the dominant production mechanism of the diffuse high-energy $\gamma$-rays in the Galactic plane, which have been measured by the Fermi-Large Area Telescope (Fermi-LAT; Ackermann et al. 2012).

In the GALPROP-based (Vladimirov et al. 2011) conventional model of Galactic diffuse $\gamma$-ray production, CRs are accelerated in a distribution of sources such as supernova remnants. They propagate diffusively in the interstellar medium producing $\gamma$-rays and neutrinos via interactions with the interstellar radiation field and interstellar gas. The interstellar radiation field is weakly constrained by Fermi-LAT $\gamma$-ray data and interstellar gas is constrained by both Fermi-LAT $\gamma$-ray data and radio measurements of $\mathrm{CO}$ and $\mathrm{HI}$ line intensities. The CR population model itself is normalized to local measurements taken at Earth. The GALPROP model parameters are tuned to

$\overline{100}$ Earthquake Research Institute, University of Tokyo, Bunkyo, Tokyo 1130032, Japan. achieve optimal agreement between Fermi-LAT (Ackermann et al. 2012) data and the direction-dependent prediction given by integrating expected $\gamma$-ray yields along the line of sight from Earth. The neutral pion decay component estimated by the conventional model should be accompanied by a neutrino flux from charged pion decay.

The conventional model, however, underpredicts the $\gamma$-ray flux above $10 \mathrm{GeV}$ in the inner Galaxy (Ackermann et al. 2012). The KRA $\gamma$ models (Gaggero et al. 2015a, 2015b, 2017) address this issue using a radially dependent model for the CR diffusion coefficient and the advective wind. The primary CR spectrum assumed within the $\mathrm{KRA}_{\gamma}$ models has an exponential cutoff at a certain energy. In order to bracket measurements by KASCADE (Antoni et al. 2005) and KASCADE-Grande (Apel et al. 2013) in the $[100 \mathrm{TeV}, 100 \mathrm{PeV}]$ and $[10 \mathrm{PeV}, 2000 \mathrm{PeV}]$ energy ranges, respectively, while maintaining agreement with proton and helium measurements by CREAM (Ahn et al. 2010), cutoffs at 5 and $50 \mathrm{PeV}$ per nucleon are considered. The resulting models are referred to as $\mathrm{KRA}_{\gamma}^{5}$ and $\mathrm{KRA}_{\gamma}^{50}$, respectively. The direction dependence of the energy-integrated $\mathrm{KRA}_{\gamma}^{5}$ neutrino 


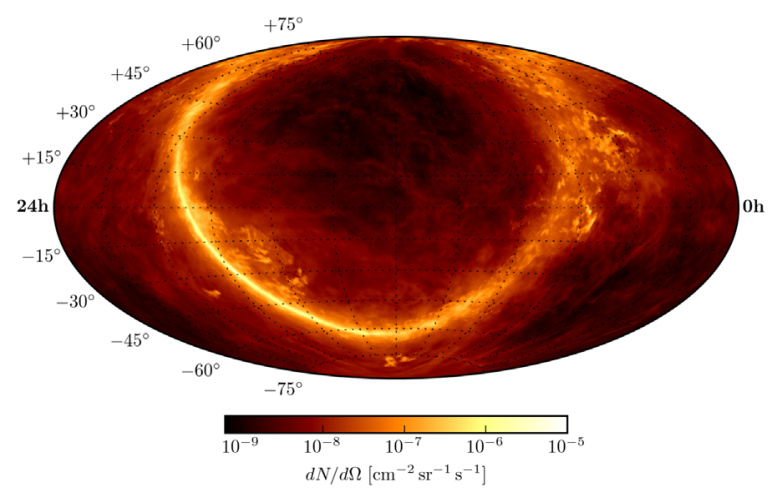

Figure 1. Neutrino flux per unit of solid angle of the $\mathrm{KRA}_{\gamma}^{5}$ model (Gaggero et al. 2015a), shown as a function of direction in equatorial coordinates (Hammer projection).

flux prediction is shown in Figure 1. Compared to the conventional model of the Galactic diffuse emission, the KRA models predict modified spectra and enhanced overall $\gamma$-ray and neutrino fluxes in the southern sky, especially in the central ridge where a hardening of the $\mathrm{CR}$ spectra is reproduced. Hence, neutrinos offer a unique opportunity to independently test the model assumptions of Galactic $\mathrm{CR}$ production and transport, accessing energies far beyond the reach of current $\gamma$-ray experiments.

The $\mathrm{KRA}_{\gamma}$ predictions have already been tested separately with Astronomy with a Neutrino Telescope and Abyss environmental RESearch (ANTARES; Albert et al. 2017) and IceCube (Aartsen et al. 2017a) data. ANTARES and IceCube

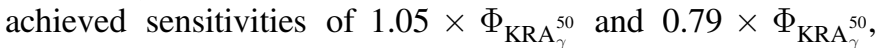
respectively; both analyses obtained $90 \%$ confidence level (CL) upper limits of $1.2 \times \Phi_{\mathrm{KRA}_{\gamma}^{50}}$. ANTARES additionally examined the $5 \mathrm{PeV}$ cutoff model, obtaining a sensitivity of $1.4 \times \Phi_{\mathrm{KRA}_{\gamma}^{5}}$ and an upper limit of $1.1 \times \Phi_{\mathrm{KRA}_{\gamma}^{5}}$ due to an underfluctuation of the fitted signal flux in the track channel.

This Letter presents a combination of these two maximumlikelihood analyses exploiting the advantageous field of view of ANTARES as well as the high statistics of IceCube.

\section{Detectors and Data Samples}

The IceCube Neutrino Observatory (Aartsen et al. 2017b) is located at the South Pole between 1.45 and $2.45 \mathrm{~km}$ below the surface of the ice. It consists of 5160 photomultiplier tubes (PMTs) instrumenting one cubic kilometer of ice. The ANTARES neutrino telescope (Ageron et al. 2011) consists of 885 PMTs deployed in the Mediterranean sea, $40 \mathrm{~km}$ off the coast of Toulon, France. It is installed at depths between $2.01 \mathrm{~km}$ and $2.47 \mathrm{~km}$ below sea level, instrumenting a volume of $\sim 0.01 \mathrm{~km}^{3}$.

Neutrinos interacting with matter produce charged particles that generate Cerenkov light in the detectors. From the collected Cerenkov light, the energy and direction of the incoming neutrinos are reconstructed. A muon neutrino ${ }^{101}$ undergoing a charged current interaction produces a muon that can travel large distances through the medium, leading to a track event topology in the detector. Most other interactions produce a nearly spherical shower event topology. In this

$\overline{101}$ In the following, particles also refer to the corresponding anti-particles.

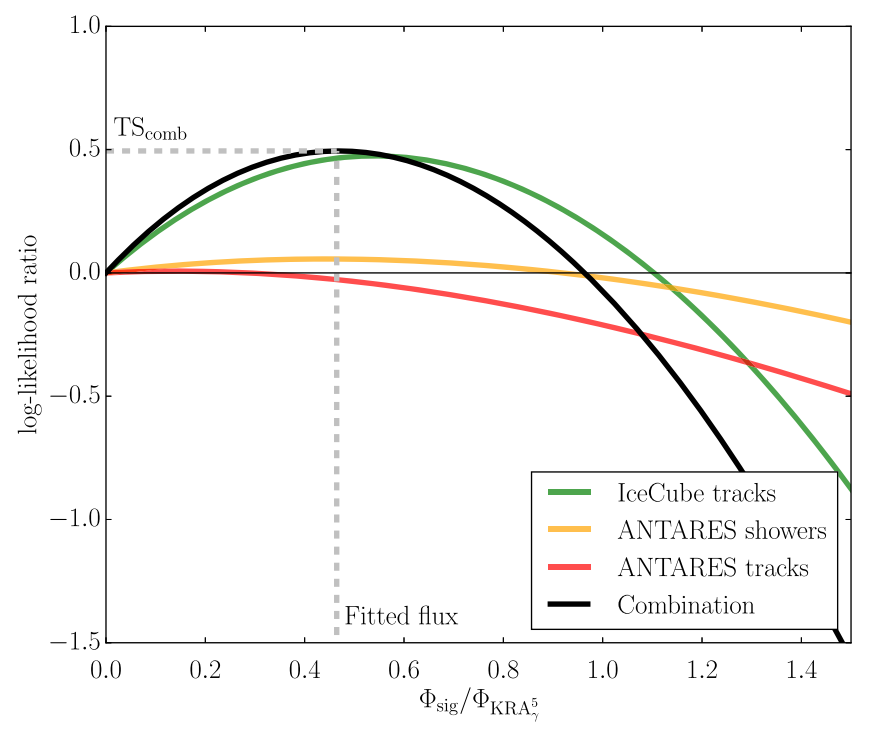

Figure 2. Combination of the log-likelihood ratio curves and fitting of the flux on the combined test statistic. These curves correspond to the unblinded data using the likelihood for the $\mathrm{KRA}_{\gamma}^{5}$ model.

analysis, ANTARES events of both topologies are used, while only track events are taken from IceCube data.

The ANTARES event sample used in this Letter includes the one used in Albert et al. (2017) extended by the data collected in 2016. These data use the most recent offlinereconstructed data set, incorporating dedicated calibrations of positioning, timing, and efficiency (Adrián-Martínez et al. 2012). The sample is taken from a total of 2780 days of detector livetime, over a total of 10 calendar years. Part of the sample was collected with partially completed detector configurations. Here, 218 shower(-like) events are selected, while 2.6 signal events are expected from the $\mathrm{KRA}_{\gamma}^{5}$ model. For these signal events we have a median angular resolution of 2.4 . The track selection includes 7,850 events, with 10.2 signal events expected to have an angular resolution of 0.5 . The energy ranges including $90 \%$ of signal events are $[2.1 \mathrm{TeV}, 150 \mathrm{TeV}]$ for showers and $[360 \mathrm{GeV}, 130 \mathrm{TeV}]$ for tracks.

The IceCube seven-year track selection used in this analysis is detailed by Aartsen et al. (2017c). It results in a total of 730,130 events with 191 events expected from the $\mathrm{KRA}_{\gamma}^{5}$ model. The data set was collected over a total of 2431 days of detector livetime, some of which took place during the construction phase of the detector. The IceCube signal events are expected to have median angular resolution of $0.8^{\circ}$. The energy range containing $90 \%$ of the expected signal events is [390 GeV, $110 \mathrm{TeV}]$.

The energy range in which the combined analysis is valid is [ $90 \mathrm{GeV}, 300 \mathrm{TeV}]$. This range is defined as containing $90 \%$ of the sensitivity. It is calculated by finding the low- and highenergy thresholds where removing simulated signal events outside of these values worsens the sensitivity by $5 \%$ each.

\section{Search Method}

The present analysis uses an unbinned likelihood ratio test. The likelihood functions for each sample-ANTARES tracks, 


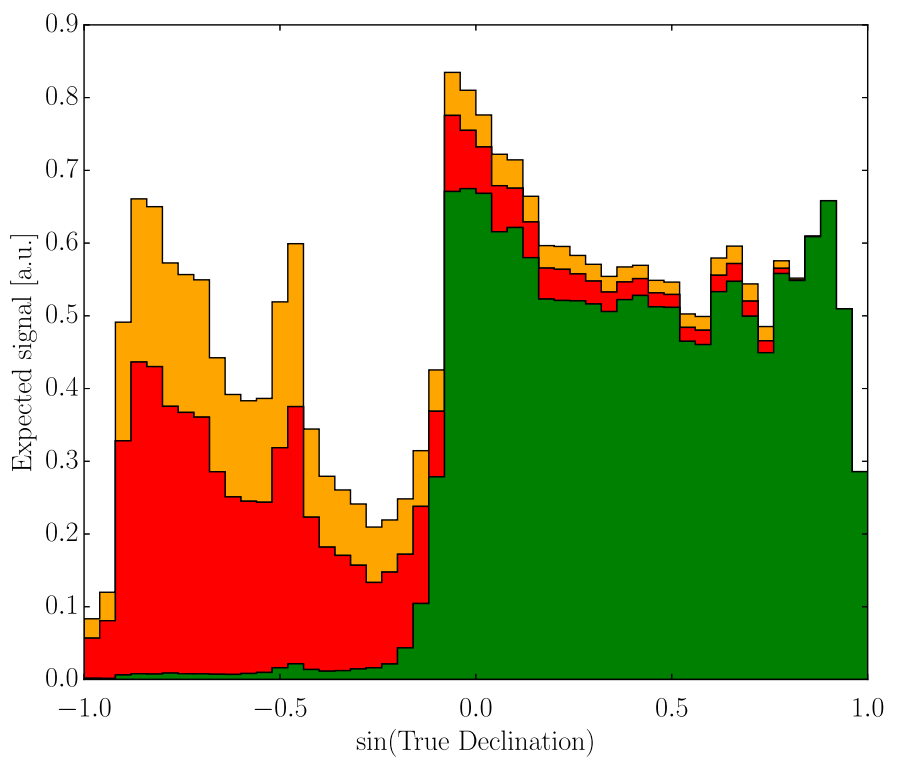

(a)

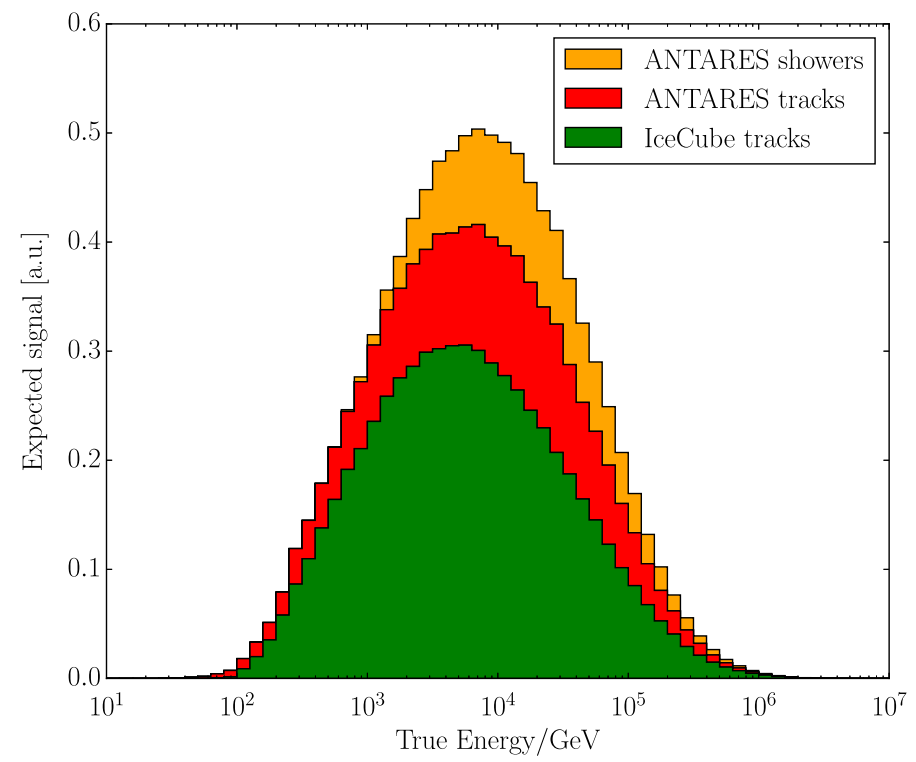

(b)

Figure 3. Stacked histograms (i.e., every bin shows the fractional contribution of every sample summed on top of each other) of the signal expected from the KRA 5 model as function of the declination (a) and energy (b) Monte Carlo truth. The colored area of each histogram represents the relative contribution to the sensitivity of this event sample. The relative contribution to the sensitivity is defined as the difference in the sensitivity flux resulting from the addition of a certain event sample divided by the combined sensitivity flux.

Table 1

Sensitivities and Results of the Analysis on the KRA $\gamma$ Models with the 5 and $50 \mathrm{PeV}$ Cutoffs

\begin{tabular}{|c|c|c|c|c|c|c|}
\hline \multirow{2}{*}{ Energy Cutoff } & \multicolumn{3}{|c|}{ Sensitivity $\left[\Phi_{\mathrm{KRA}_{\gamma}}\right]$} & \multirow{2}{*}{$\begin{array}{c}\text { Fitted Flux } \\
{\left[\Phi_{\mathrm{KRA}_{\gamma}}\right]}\end{array}$} & \multirow{2}{*}{$\begin{array}{c}p \text {-value } \\
{[\%]}\end{array}$} & \multirow{2}{*}{$\begin{array}{c}\text { Upper Limit (UL) at } 90 \% \mathrm{CL} \\
{\left[\Phi_{\mathrm{KRA}_{\gamma}}\right]}\end{array}$} \\
\hline & Combined & ANTARES & IceCube & & & \\
\hline$\overline{5 \mathrm{PeV}}$ & 0.81 & 1.21 & 1.14 & 0.47 & 29 & 1.19 \\
\hline
\end{tabular}

ANTARES showers, and IceCube tracks—are defined as

$$
\begin{aligned}
\mathcal{L}_{\text {sig }+ \text { bkg }}\left(n_{\text {sig }}\right)= & \prod_{i}\left[\frac{n_{\text {sig }}}{N} \cdot \mathcal{S}\left(E_{i}, \alpha_{i}, \delta_{i}\right)\right. \\
& \left.+\left(1-\frac{n_{\text {sig }}}{N}\right) \cdot \mathcal{B}\left(E_{i}, \delta_{i}\right)\right],
\end{aligned}
$$

where $N$ is the total number of events, $n_{\text {sig }}$ is the number of signal events, and $\mathcal{S}$ is the signal probability density function (PDF) for an event $i$ at the equatorial coordinates $\left(\alpha_{i}, \delta_{i}\right)$ with energy $E_{i}$. It is obtained from Monte Carlo simulations of the detectors with the model flux as input, and is proportional to the expected signal rate at a given reconstructed energy and direction. $\mathcal{B}$ is the PDF of the background.

Minor differences in the original, separate ANTARES and IceCube PDF constructions are preserved in this Letter. For IceCube tracks, the background term $\mathcal{B}$ comes from the data with a correction for the signal contamination expected for $n_{\text {sig }}$ signal events (Aartsen et al. 2017a). For the ANTARES samples, this is approximated by ignoring the signal correction term (Albert et al. 2017). In addition, the IceCube signal PDF accounts for the estimated point-spread function of each event, while average point-spread functions are used for track and shower ANTARES events.

In order to account for the different acceptances of each sample as well as any bias in the fitted signal

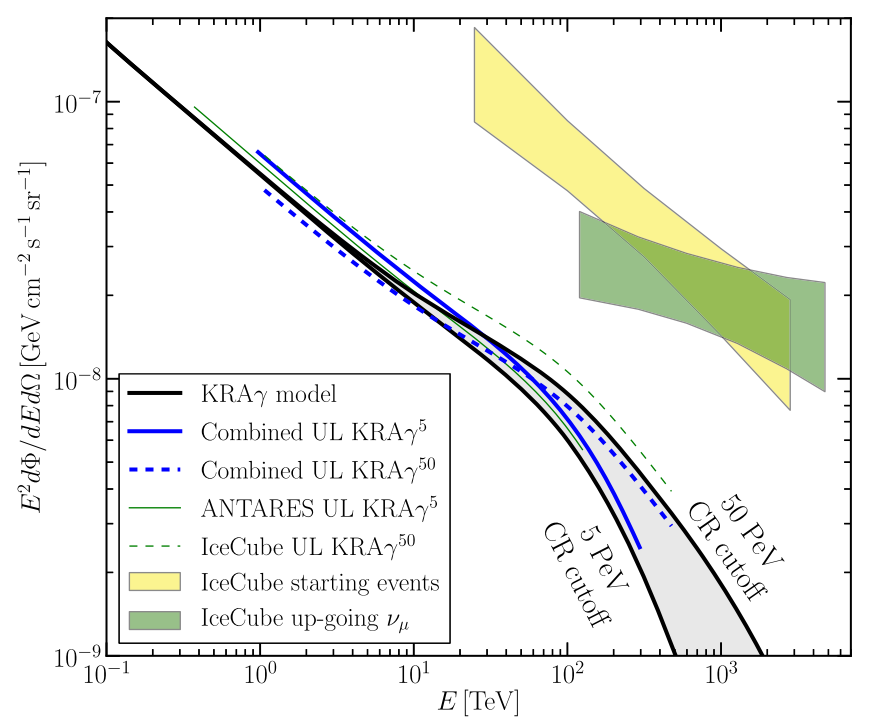

Figure 4. Combined ULs at $90 \%$ confidence level (blue lines) on the three-flavor neutrino flux of the $\mathrm{KRA}_{\gamma}$ model with the 5 and $50 \mathrm{PeV}$ cutoffs (black lines). The boxes represent the diffuse astrophysical neutrino fluxes measured by IceCube using an isotropic flux template with starting events (yellow) and upgoing tracks (green).

normalization, we forward-fold the signal flux $\Phi_{\text {sig }}$ into the individual likelihoods using a response function obtained from simulated pseudo-experiments. 
Then the combined likelihood is simply the product over the per-sample likelihoods. The combined test statistic is the loglikelihood ratio evaluated for that $\Phi_{\text {sig }}$, which maximizes the combined likelihood

$$
\mathrm{TS}_{\mathrm{comb}}=\max _{\Phi_{\mathrm{sig}}}\left\{\sum_{\text {sample }} \ln \left[\frac{\mathcal{L}_{\mathrm{sig}+\mathrm{bkg}}\left(\Phi_{\mathrm{sig}}\right)}{\mathcal{L}_{\mathrm{bkg}}}\right]\right\},
$$

where $\mathrm{TS}_{\mathrm{comb}}$ is the combined test statistic, and $\mathcal{L}_{\text {bkg }}=\mathcal{L}_{\text {sig }+ \text { bkg }}\left(\Phi_{\text {sig }}=0\right)$ is the likelihood to have only background and the sum runs over the event samples. This is illustrated in Figure 2 with the combined log-likelihood ratio and $\mathrm{TS}_{\mathrm{comb}}$ fit for the $\mathrm{KRA}_{\gamma}^{5}$ model.

The combined and independent sensitivities are summarized in Table 1 . They are defined as the median upper limit. ${ }^{102}$ The combination is not only a way to exploit more data with different systematics, but also an opportunity to benefit from the complementarity of the two detectors. While IceCube has much higher statistics than ANTARES, we show in Figure 3(a) that ANTARES offers enhanced sensitivity in the southern sky where a larger flux is expected. This favorable view is coupled with relatively better angular resolution for ANTARES than IceCube. In Figure 3(b) we show that while IceCube can in principle detect higher energy events compared to ANTARES, the direction-dependent model spectra studied here result in similar energy ranges being tested by both detectors. Overall, the relative contribution of IceCube to the sensitivity is $61 \%$; for ANTARES tracks and showers the relative contributions are $25 \%$ and $14 \%$, respectively.

\section{Results and Discussion}

This analysis combines seven years of IceCube tracks and ten years of ANTARES tracks and showers using a likelihood ratio test. The results are summarized in Table 1. Systematic uncertainties on the ANTARES detection efficiency (due to the uncertainty on the acceptance of the ANTARES PMTs) are included in the analysis as in the paper by Albert et al. (2017). As described by Aartsen et al. (2017c), systematic uncertainties in the modeling of the Antarctic ice and the optical module efficiency lead to an uncertainty on the IceCube detection efficiency of at most $11 \%$, which is not included here.

The maximum-likelihood estimate yields a non-zero diffuse Galactic neutrino flux for both models with a p-value of $29 \%$ for $\mathrm{KRA}_{\gamma}^{5}$ and $26 \%$ for $\mathrm{KRA}_{\gamma}^{50}$. As neither of these results is statistically significant, we place upper limits on both model normalizations. The $\mathrm{KRA}_{\gamma}^{50}$ model is constrained at the $90 \%$ CL (with an upper limit of $0.9 \times \Phi_{\mathrm{KRA}^{50}}$ ), while the $\mathrm{KRA}_{\gamma}^{5}$ model is not yet constrained by our analysis. This was expected as the $50 \mathrm{PeV}$ cutoff represents an extreme tuning of the acceleration parameters for the Galactic CRs, while the $5 \mathrm{PeV}$ cutoff in light $\mathrm{CR}$ can be considered a more reliable case for the Galactic accelerators.

Figure 4 represents the combined upper limits in comparison to the all-flavor full-sky energy spectrum of the $\mathrm{KRA}_{\gamma}$ models as well as the previous IceCube and ANTARES upper limits. The present upper limit on the $5 \mathrm{PeV}$ model is higher than the previously published upper limit for ANTARES alone, although the sensitivity is much better. This is due to the

\footnotetext{
102 It is defined as the average upper limit in the ANTARES-only analysis (Albert et al. 2017). This and the addition of 2016 data account for the difference in the ANTARES sensitivities.
}

overfluctuation observed in the IceCube data sample as well as the difference in the definition of the test statistic. In the ANTARES stand-alone analysis it was the sum of the shower and track test statistics, computed independently, instead of computing one test statistic from the combined log-likelihood ratio curve (Equation (2)).

The results presented here provide for the first time a combined constraint on diffuse Galactic neutrino emission by IceCube and ANTARES. The limit on the $\mathrm{KRA}_{\gamma}$ model with $50 \mathrm{PeV}$ cutoff extends the energy range of the constraint on the model from $10 \mathrm{GeV}$ with Fermi-LAT up to hundreds of TeV. Based on the limit on the $\mathrm{KRA}_{\gamma}^{5}$-model, this analysis limits the total flux contribution of diffuse Galactic neutrino emission to the total astrophysical signal reported by Aartsen et al. (2015) to $8.5 \%$. In the future, the sensitivity of this analysis can be further improved by including IceCube showers (Aartsen et al. 2017d). This will allow for a powerful test of the $\mathrm{KRA}_{\gamma}^{5}$ model, thereby constraining the diffusion mechanisms, the maximal energy injected by supernova remnants and the Galactic gas distributions considered in the model.

ANTARES acknowledges the financial support of the funding agencies: Centre National de la Recherche Scientifique (CNRS), Commissariat à l'énergie atomique et aux énergies alternatives (CEA), Commission Européenne (FEDER fund and Marie Curie Program), Institut Universitaire de France (IUF), IdEx program and UnivEarthS Labex program at Sorbonne Paris Cité (ANR-10-LABX-0023 and ANR-11IDEX-0005-02), Labex OCEVU (ANR-11-LABX-0060) and the $A^{*}$ MIDEX project (ANR-11-IDEX-0001-02), Région Îlede-France (DIM-ACAV), Région Alsace (contrat CPER), Région Provence-Alpes-Côte d'Azur, Département du Var and Ville de La Seyne-sur-Mer, France; Bundesministerium für Bildung und Forschung (BMBF), Germany; Istituto Nazionale di Fisica Nucleare (INFN), Italy; Nederlandse organisatie voor Wetenschappelijk Onderzoek (NWO), the Netherlands; Council of the President of the Russian Federation for young scientists and leading scientific schools supporting grants, Russia; National Authority for Scientific Research (ANCS), Romania; Ministerio de Economía y Competitividad (MINECO): Plan Estatal de Investigación (refs. FPA201565150-C3-1-P, -2-P, and -3-P, (MINECO/FEDER)), Severo Ochoa Centre of Excellence and MultiDark Consolider (MINECO), and Prometeo and Grisolía programs (Generalitat Valenciana), Spain; Ministry of Higher Education, Scientific Research and Professional Training, Morocco. We also acknowledge the technical support of Ifremer, AIM, and Foselev Marine for the sea operation and the CC-IN2P3 for the computing facilities.

The IceCube Neutrino Observatory acknowledges support from the following agencies: USA-U.S. National Science Foundation-Office of Polar Programs, U.S. National Science Foundation-Physics Division, Wisconsin Alumni Research Foundation, Center for High Throughput Computing (CHTC) at the University of Wisconsin-Madison, Open Science Grid (OSG), Extreme Science and Engineering Discovery Environment (XSEDE), U.S. Department of Energy-National Energy Research Scientific Computing Center, Particle astrophysics research computing center at the University of Maryland, Institute for Cyber-Enabled Research at Michigan State University, and Astroparticle physics computational facility at Marquette University; Belgium_-Funds for Scientific Research (FRS-FNRS 
and FWO), FWO Odysseus and Big Science programmes, and Belgian Federal Science Policy Office (Belspo); GermanyBundesministerium für Bildung und Forschung (BMBF), Deutsche Forschungsgemeinschaft (DFG), Helmholtz Alliance for Astroparticle Physics (HAP), Initiative and Networking Fund of the Helmholtz Association, Deutsches Elektronen Synchrotron (DESY), and High Performance Computing cluster of the RWTH Aachen; Sweden-Swedish Research Council, Swedish Polar Research Secretariat, Swedish National Infrastructure for Computing (SNIC), and Knut and Alice Wallenberg Foundation; Australia-Australian Research Council; Canada-Natural Sciences and Engineering Research Council of Canada, Calcul Québec, Compute Ontario, Canada Foundation for Innovation, WestGrid, and Compute Canada; Denmark-Villum Fonden, Danish National Research Foundation (DNRF); New ZealandMarsden Fund; Japan-Japan Society for Promotion of Science (JSPS) and Institute for Global Prominent Research (IGPR) of Chiba University; Korea-National Research Foundation of Korea (NRF); Switzerland-Swiss National Science Foundation (SNSF).

The IceCube Collaboration designed, constructed, and now operates the IceCube Neutrino Observatory. Data processing and calibration, Monte Carlo simulations of the detector and of theoretical models, and data analyses were performed by a large number of collaboration members, who also discussed and approved the scientific results presented here. It was reviewed by the entire collaboration before publication, and all authors approved the final version of the manuscript.

The main authors of this manuscript were Jon Dumm, Timothée Grégoire, Christian Haack, and Michael Richman.

\section{References}

Aartsen, M. G., et al. 2017d, ApJ, 846, 136

Aartsen, M. G., Abraham, K., Ackermann, M., et al. 2015, ApJ, 809, 98

Aartsen, M. G., Abraham, K., Ackermann, M., et al. 2017c, ApJ, 835, 151

Aartsen, M. G., Ackermann, M., Adams, J., et al. 2017a, ApJ, 849, 67

Aartsen, M. G., Ackermann, M., Adams, J., et al. 2017b, JInst, 12, P03012

Ackermann, M., Ajello, M., Atwood, W. B., et al. 2012, ApJ, 750, 3

Adrián-Martínez, S., Ageron, M., Aguilar, J. A., et al. 2012, JInst, 7 , T08002

Ageron, M., Aguilar, J. A., Al Samarai, I., et al. 2011, Nucl. Instrum. Meth, A656, 11

Ahn, H. S., Allison, P., Bagliesi, M. G., et al. 2010, ApJL, 714, L89

Albert, A., André, M., Anghinolfi, M., et al. 2017, PhRvD, 96, 062001

Antoni, T., Apel, W. D., Badea, A. F., et al. 2005, APh, 24, 1

Apel, W. D., Arteaga-Velàzquez, J. C., Bekk, K., et al. 2013, APh, 47, 54

Gaggero, D., Grasso, D., Marinelli, A., Taoso, M., \& Urbano, A. 2017, PhRvL, 119,031101

Gaggero, D., Grasso, D., Marinelli, A., Urbano, A., \& Valli, M. 2015a, ApJL, $815, \mathrm{~L} 25$

Gaggero, D., Urbano, A., Valli, M., \& Ullio, P. 2015b, PhRvD, D91, 083012

Vladimirov, A. E., Digel, S. W., Jóhannesson, G., et al. 2011, CoPhC, 182,1156 